\title{
Development and verification of a finite volume model for hydraulics over multi-layered erodible beds
}

\author{
Thomas Rowan \& Mohammed Seaid \\ Department of Engineering and Computer Sciences \\ University of Durham, Durham, England
}

\begin{abstract}
The accuracy and efficiency of a class of finite volume methods are investigated for numerical solution of morphodynamic problems in multi-layered beds. The governing equations consist of two components, namely an hydraulic part described by the shallow water equations and a sediment part described by the transport and suspended sediments equations. To allow exchange between layers in the bed we propose an equation of balance laws for the bed elevation. As a numerical solver we consider a modified Roe method in the finite volume framework. A well balanced discretization is also used for the treatment of the source terms. The method is well-balanced, non-oscillatory and suitable for both near- and far-bed simulations in sediment transport. The proposed model is verified for a dam-break problem over erodible multi-layered bed and the obtained results demonstrate it abilities to resolve the vertical features in multi-layered sediment transport problems.
\end{abstract}

\section{INTRODUCTION}

The main concern of the sediment transport (or morphodynamics) is to determine the evolution of bed levels for hydrodynamic systems such as rivers, estuaries, bays and other nearshore regions where water flows interact with the bed geometry. Example of applications include among others, beach profile changes due to severe wave climates, seabed response to dredging procedures or imposed structures, and harbour siltation. In general, suspended sediments and bed-load transport in shallow water flows are determined by the characteristics of the hydraulics flow and the properties of the suspended sediments. Thus, dynamics of the water and dynamics of the sediments must be studied using a mathematical model made of three different but dependent model variables: (i) a set of hydraulics variables defining the dynamics of the water flow, (ii) a sediment variable defining the transport and dispersion of the sediments and (iii) a topography variable defining the dynamics of the bed-load. Most existing models for this class of problems either consider the three-dimensional Navier-Stokes equations or a depth-averaged system widely known by shallow water equations for the hydrodynamics coupled to an Exner-type of equations for the bed-load, compare $[11,1,18]$ and further references are therein. The difficulties in the first approach are seen by the remeshing required to deal with moving boundaries for free-surface and bottom topography due to hydrostatic pressure and erosion/deposition forces, respec- tively. Because its high computational cost this approach is rarely used in practical applications and it is mainly kept as last resort. The drawback in the second approach lies on the failure of the coupled shallow water and Exner equations to capture vertical effects in a soil-superposed packed bed.

In the current study we present a new-coupled model for hydraulics over multi-layered erodible beds. The bed is assumed to be heterogeneous and formed with multiple layers with different soil properties. The structure of soil-superposed packed beds is given along with the total number of layers to be considered in the analysis. The governing equations consist of a coupled system of shallow water equations for the hydraulics, an Exner equation for the bedload, a transport equation for suspended sediments, and a series of exchange terms for the mass transfer between the bed layers due to erosion and/or deposition effects. These equations form a nonlinear hyperbolic system of conservation laws with source terms to be solved for the time variable, the horizontal space coordinate and the bed depth. Such practical coupled hydrodynamical and morphodynamical problems are not trivial to simulate since the soil can be heterogeneous and the topography irregular. It should be pointed out that other mathematical models for sediment transport have also been studied in [14] among others. In these systems, the shallow water equations have been coupled to an Exner-type equation for the bed-load without accounting for suspended sediments as it is shown in the current study. 
The ability to design numerical methods able to predict the hydraulics over multi-layered erodible beds has a clear mathematical and engineering relevancies. Nowadays, much effort has been devoted to develop numerical schemes for sediment transport models able to resolve all hydrodynamics and morphodynamics scales. In the current study, a class of finite volume methods is proposed for numerical simulation of transient flows involving erosion and deposition of sediments. The method consists of a predictor stage where the numerical fluxes are constructed and a corrector stage used to recover the conservation equations. The sign of the Jacobian matrix is used in the reconstruction of the numerical fluxes. Most of these techniques have been recently investigated in [2] for solving morphodynamics models without accounting for sediment transport, erosion and deposition effects. The current study presents an extension of this method to transient flows involving erosion and deposition of sediments as well as the exchange between bed layers. The proposed method also satisfies the property of well-balancing flux-gradient and source-term in the system. Results presented in this paper demonstrate high resolution of the proposed method and confirm its capability to provide accurate and efficient simulations for sediment transport by water flows including erosion and deposition effects in complex topography.

The outline of this paper is structured as follows. In section 2 we briefly present the governing equations for hydraulics over multi-layered erodible beds. The finite volume method is formulated in section 3 . This latter includes both the discretization of gradient fluxes and the treatment of source terms as well as the resolution of exchange terms between the layers. $\mathrm{Nu}-$ merical results for benchmark and novel experiments are presented in section 4 . In section 5 concluding remarks are summarized.

\section{FORMULATION OF THE PROBLEM}

Modelling hydraulics over multi-layered erodible beds requires equations for hydrodynamics, for morphodynamics and for suspended sediments. In this study we assume that the flow is almost horizontal, the vertical component of the acceleration is vanishingly small, the pressure is taken to be hydrostatic, the free-surface gravity waves are long with respect to the mean flow depth and wave amplitude, and the water-species mixture is vertically homogeneous and non-reactive. The governing equations are obtained by balancing the net inflow of mass, momentum and species through boundaries of a control volume during an infinitesimal time interval while accounting for the accumulation of mass, resultant forces and species within the control volume, compare for example [1] among others. Thus, the equations for mass conservation and momentum flux balance are given by $\frac{\partial h}{\partial t}+\frac{\partial(h u)}{\partial x} \frac{E-D}{1-p}$,

$$
\begin{array}{r}
\frac{\partial(h u)}{\partial t}+\frac{\partial}{\partial x}\left(h u^{2}+\frac{1}{2} g h^{2}\right)=-g h \frac{\partial B}{\partial x}-\frac{\left(\rho_{s}-\rho_{w}\right)}{2 \rho} g h^{2} \frac{\partial c}{\partial x} \\
-\frac{\left(\rho_{0}-\rho\right)(E-D) u}{\rho(1-p)}-g h S_{f},
\end{array}
$$

where $u$ is the depth-averaged water velocity, $h$ the water depth, $B$ the bottom topography, $g$ the gravitational acceleration, $p$ the porosity, $\rho_{w}$ the water density, $\rho_{s}$ the sediment density, $c$ is the depth-averaged concentration of the suspended sediment, $E$ and $D$ represent the entrainment and deposition terms in upward and downward directions, respectively. In (1), $\rho$ and $\rho_{0}$ are defined as the density of the watersediment mixture and the density of the saturated bed respectively

$\rho=\rho_{w}(1-c)+\rho_{s} c, \quad \rho_{0}=\rho_{w} p+\rho_{s}(1-p)$,

and $S_{f}$ is the friction slope involving the Manning roughness coefficient $n_{b}$ as

$S_{f}=\frac{n_{b}^{2} u^{2}}{h^{4 / 3}}$.

The equation for mass conservation of species is modelled by

$$
\frac{\partial(h c)}{\partial t}+\frac{\partial(h u c)}{\partial x}=E-D .
$$

To determine the entrainment and deposition terms in the above equations we assume a non-cohesive sediment and we use empirical relations reported in [7]. Thus,

$D=w\left(1-C_{a}\right)^{m} C_{a}$,

where $w$ is deposition coefficient as experimentally measured $[6,19,17], d$ the averaged diameter of the sediment particle, $m$ an exponent indicating the effects of hindered settling due to high sediment concentrations, and $C_{a}$ the near-bed volumetric sediment concentration, $C_{a}=\alpha_{c} c$, with $\alpha_{c}$ is a coefficient larger than unity. To ensure that the near-bed concentration does not exceed $(1-p)$, the coefficient $\alpha_{c}$ is computed by [10]

$\alpha_{c}=\min \left(2, \frac{1-p}{c}\right)$.

For the entrainment of a cohesive material the following relation is used

$E= \begin{cases}\varphi \frac{\theta-\theta_{c}}{h} u d^{-0.2}, & \text { if } \theta \geq \theta_{c} \\ 0, & \text { otherwise }\end{cases}$ 
where $\varphi$ is a coefficient to control the erosion forces, $\theta_{c}$ is a critical value of Shields parameter for the initiation of sediment motion and $\theta$ is the Shields coefficient defined by

$\theta=\frac{u_{*}^{2}}{s g d}$

and $u_{*}$ as the friction velocity defined as

$u_{*}^{2}=\sqrt{\frac{g n_{b}^{2}}{h^{1 / 3}}}|u|$.

In (6), $s$ is the submerged specific gravity of sediment given by

$s=\frac{\rho_{s}}{\rho_{w}}-1$.

To update the bedload, we consider the Exner equation

$\frac{\partial B}{\partial t}=-\frac{E-D}{1-p}$.

Most sediment transport models are empirical to differing extents as they have been derived from experiments. More mathematical and physical aspects of the problem are considered in $[8,9]$ among others. In these references, the bed topography is assumed to be homogeneous formed with a single soil. In the current study we are interested in hydraulic problems over multi-layered erodible beds in which the topography is heterogeneous and formed with multiple soils with different properties, see Figure 1 for an illustration. This requires different sediment parameters to be accounted for in the friction slope, entrainment and deposition terms. Here, the single-layer bed $[0, B]$ is decomposed into multi-layered beds $\left[B_{k-1}, B_{k}\right]$, with $k=1,2, \ldots, M, B_{0}=B$ and $B_{M}=0$. Notice that this decomposition is known a priori and the total number of layers in the bed $M$ is also given. At this stage all the sediment properties should be defined depending on each layer in the bed. For example, the porosity is defined as

$p(z)=p_{k}, \quad$ if $\quad z \in\left[B_{k-1}, B_{k}\right], \quad k=1,2, \ldots, M$,

with a similar equation for $\rho_{s}, d, n_{b}, \varphi, \theta_{c}$ and $f$. In (8), $p_{k}$ is a given porosity coefficient associated with each soil type used in the associated layer $\left[B_{k-1}, B_{k}\right]$. By taking into consideration this variation in the bed, the governing equations (1) become bed-dependent in which an extra coordinate $z$ is added to the spacial coordinate $x$ and the time coordinate $t$.

For simplicity in presentation, the equations (1) can be rearranged in vector form for a system of conservation laws with source terms as

$\frac{\partial \mathbf{W}}{\partial t}+\frac{\partial \mathbf{F}(\mathbf{W})}{\partial x}=\mathbf{S}(\mathbf{W})+\mathbf{Q}(\mathbf{W})$

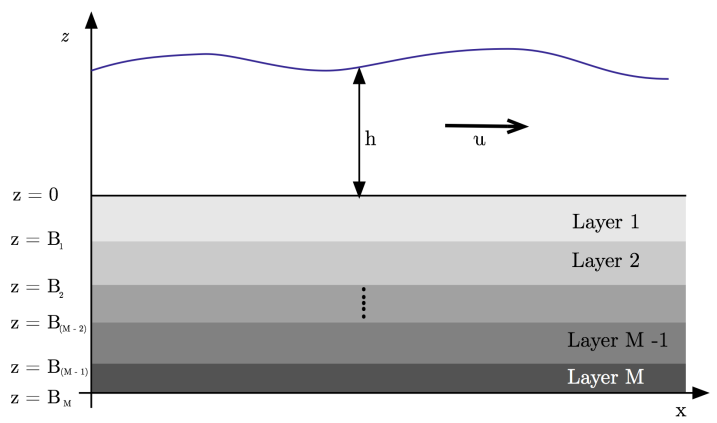

Figure 1: Illustration of sediment transport in shallow water flows over a multi-layered bed.

where

$\mathbf{W}=\left(\begin{array}{c}h \\ h u \\ h c \\ B\end{array}\right), \quad \mathbf{F}(\mathbf{W})=\left(\begin{array}{c}h u \\ h u^{2}+\frac{1}{2} g h^{2} \\ h u c \\ 0\end{array}\right)$,

$\mathbf{S}(\mathbf{W})=\left(\begin{array}{c}0 \\ -g h \frac{\partial Z}{\partial x}-\frac{\left(\rho_{s}-\rho_{w}\right)}{2 \rho} g h^{2} \frac{\partial c}{\partial x} \\ 0 \\ 0\end{array}\right), \mathbf{Q}(\mathbf{W})=\left(\begin{array}{c}\frac{E-D}{1-p} \\ -\frac{\left(\rho_{0}-\rho\right)(E-D) u}{\rho(1-p)}-g h S_{f} \\ E-D \\ -\frac{E-D}{1-p}\end{array}\right)$.

The system (9) can be rearranged in a nonconservative form as

$\frac{\partial \mathbf{W}}{\partial t}+\mathcal{A}(\mathbf{W}) \frac{\partial \mathbf{W}}{\partial x}=\mathcal{Q}(\mathbf{W})$,

where

$\mathcal{A}(\mathbf{W})=\left(\begin{array}{cccc}0 & 1 & 0 & 0 \\ g h-u^{2}-\frac{\left(\rho_{s}-\rho_{w}\right)}{2 \rho} g h c & 2 u & \frac{\left(\rho_{s}-\rho_{w}\right)}{2 \rho} g h & g h \\ -u c & c & u & 0 \\ 0 & 0 & 0 & 0\end{array}\right)$.

It is evident that the system (9) is of hyperbolic type with the four real and distinct eigenvalues $\lambda_{k}(k=$ $1,2,3,4)$ of the matrix $\mathcal{A}$ are given by

$\lambda_{1}=0, \quad \lambda_{2}=u, \quad \lambda_{3}=u-\sqrt{g h}, \quad \lambda_{4}=u+\sqrt{g h}$,

and their associated eigenvectors are

$e_{1}=\left(\begin{array}{c}-g h \\ 0 \\ -g h c \\ g h-u^{2}\end{array}\right), e_{2}=\left(\begin{array}{c}\frac{\rho_{s}-\rho_{w}}{2 \rho} \\ \frac{\rho_{s}-\rho_{w}}{2 \rho} u \\ \frac{\rho_{s}-\rho_{w}}{2 \rho} c-1 \\ 0\end{array}\right)$, 
$e_{3}=\left(\begin{array}{c}1 \\ u-\sqrt{g h} \\ c \\ 0\end{array}\right), e_{4}=\left(\begin{array}{c}1 \\ u+\sqrt{g h} \\ c \\ 0\end{array}\right)$.

Note that the considered sediment transport system (9) have to be solved in a horizontal spatial domain for the variable $x$, for a vertical spatial domain for the variable $z$, and for a time interval for the variable $t$.

\section{THE NUMERICAL METHOD}

To discretize the system (9) in the $x$ dimension we discretize the spatial domain into control volumes $\left[x_{i-1 / 2}, x_{i+1 / 2}\right]$ with same length $\Delta x$ for sake of simplicity. We also divide the time interval into subintervals $\left[t_{n}, t_{n+1}\right]$ with uniform size $\Delta t$. Here, $t_{n}=$ $n \Delta t, x_{i-1 / 2}=i \Delta x$ and $x_{i}=(i+1 / 2) \Delta x$ is the centre of the control volume. Following the standard finite volume formulation, we integrate the equation (9) with respect to time and space over the domain $\left[t_{n}, t_{n+1}\right] \times\left[x_{i-1 / 2}, x_{i+1 / 2}\right]$ to obtain the following discrete equation

$\mathbf{W}_{i}^{n+1}=\mathbf{W}_{i}^{n}-\frac{\Delta t}{\Delta x}\left(\mathbf{F}_{i+1 / 2}^{n}-\mathbf{F}_{i-1 / 2}^{n}\right)+\Delta t \mathbf{S}_{i}^{n}+\Delta t \mathbf{Q}\left(\mathbf{W}_{i}^{n}\right)$,

where $\mathbf{W}_{i}^{n}$ is the space average of the solution $\mathbf{W}$ in the control volume $\left[x_{i-1 / 2}, x_{i+1 / 2}\right]$ at time $t_{n}$ i.e.,

$\mathbf{W}_{i}^{n}=\frac{1}{\Delta x} \int_{x_{i-1 / 2}}^{x_{i+1 / 2}} \mathbf{W}\left(t_{n}, x\right) d x$, content...

and $\mathcal{F}_{i \pm 1 / 2}^{n}=\mathbf{F}\left(\mathbf{W}_{i \pm 1 / 2}^{n}\right)$ are the numerical fluxes at $x=x_{i \pm 1 / 2}$ and time $t_{n}$. To deal with the source terms $\mathcal{S}$ and $\mathcal{Q}$, a standard splitting procedure is employed for (12) as

$$
\begin{aligned}
\mathbf{W}_{i}^{*} & =\mathbf{W}_{i}^{n}+\Delta t \mathbf{Q}\left(\mathbf{W}_{i}^{n}\right), \\
\mathbf{W}_{i}^{n+1} & =\mathbf{W}_{i}^{*}-\frac{\Delta t}{\Delta x}\left(\mathbf{F}_{i+1 / 2}^{n}-\mathbf{F}_{i-1 / 2}^{n}\right)+\Delta t \mathbf{S}_{i}^{n} .
\end{aligned}
$$

The spatial discretization of the equation (14) is complete when a numerical reconstruction of the fluxes $\mathcal{F}_{i \pm 1 / 2}^{n}$ and source term $\mathcal{S}_{i}^{n}$ is selected. In general, this reconstruction requires a solution of Riemann problems at the interfaces $x_{i \pm 1 / 2}$. In order to avoid difficulties associated with the Riemann solvers and reconstruct an approximation of $\mathbf{W}_{i+1 / 2}^{n}$, we adapt a finite volume non-homogeneous Riemann solver (hereafter referred to as FV method) proposed in [12]. The FV method has also been extended to morphodynamics problems in $[3,2]$. In the current study we examine the performance of the FV method for solving dambreak flows over erodible sediment beds. Hence, applied to the first step in the splitting (14), the FV results in a predictor-corrector scheme of the form

$\mathbf{W}_{i+1 / 2}^{n}=\frac{1}{2}\left(\mathbf{W}_{i+1}^{*}+\mathbf{W}_{i}^{*}\right)-\frac{1}{2} \operatorname{sgn}\left[\mathcal{A}\left(\nu\left(\overline{\mathbf{W}}_{i}, \overline{\mathbf{W}}_{i+1}\right)\right)\right]\left(\mathbf{W}_{i+1}^{*}-\mathbf{W}_{i}^{*}\right)$,

$$
\mathbf{W}_{i}^{n+1}=\mathbf{W}_{i}^{n}-\frac{\Delta t}{\Delta x}\left(\mathbf{F}\left(\mathbf{W}_{i+1 / 2}^{n}\right)-\mathbf{F}\left(\mathbf{W}_{i-1 / 2}^{n}\right)\right)+\Delta t \mathbf{S}_{i}^{n}+\Delta t \mathbf{Q}_{i}^{n},
$$

where the averaged state $\nu\left(\overline{\mathbf{W}}_{i}, \overline{\mathbf{W}}_{i+1}\right)$ is calculated as

$\nu\left(\overline{\mathbf{W}}_{i}, \overline{\mathbf{W}}_{i+1}\right)=\left(\begin{array}{c}\bar{h}_{i+\frac{1}{2}} \\ \bar{u}_{i+\frac{1}{2}} \\ \bar{c}_{i+\frac{1}{2}} \\ \bar{B}_{i+\frac{1}{2}}\end{array}\right)=\left(\begin{array}{c}\frac{h_{i}+h_{i+1}}{2} \\ \frac{\sqrt{h_{i}} u_{i}+\sqrt{h_{i+1}} u_{i+1}}{\sqrt{h_{i}}+\sqrt{h_{i+1}}} \\ \frac{\sqrt{h_{i}} c_{i}+\sqrt{h_{i+1}} c_{i+1}}{\sqrt{h_{i}}+\sqrt{h_{i+1}}} \\ \frac{B_{i}+B_{i+1}}{2}\end{array}\right)$,

and the sign matrix is given by

$\operatorname{sgn}\left[\mathcal{A}\left(\nu\left(\overline{\mathbf{W}}_{i}, \overline{\mathbf{W}}_{i+1}\right)\right)\right]=\mathcal{R}\left(\overline{\mathbf{W}}_{i+\frac{1}{2}}\right)\left|\Lambda\left(\overline{\mathbf{W}}_{i+\frac{1}{2}}\right)\right|^{-1} \Lambda\left(\overline{\mathbf{W}}_{i+\frac{1}{2}}\right) \mathcal{R}^{-1}\left(\overline{\mathbf{W}}_{i+\frac{1}{2}}\right)$.

This matrix can be explicitly expressed using the associated eigenvalues from (11). Next we shall discuss the determination of the sign matrix in (15) and we also provide details on the treatment of source terms $\mathbf{S}_{i}^{n}$.

The determination of the sign matrix $\operatorname{sgn}[\mathcal{A}(\overline{\mathbf{W}})]$ is carried out using the eigenvalues in (11) as

$\operatorname{sgn}[\mathcal{A}(\overline{\mathbf{W}})]=\mathcal{R}(\overline{\mathbf{W}}) \operatorname{sgn}[\Lambda(\overline{\mathbf{W}})] \mathcal{R}^{-1}(\overline{\mathbf{W}})$,

where

$\operatorname{sgn}[\Lambda(\overline{\mathbf{W}})]=\left(\begin{array}{cccc}\operatorname{sgn}\left(\bar{\lambda}_{1}\right) & 0 & 0 & 0 \\ 0 & \operatorname{sgn}\left(\bar{\lambda}_{2}\right) & 0 & 0 \\ 0 & 0 & \operatorname{sgn}\left(\bar{\lambda}_{3}\right) & 0 \\ 0 & 0 & 0 & \operatorname{sgn}\left(\bar{\lambda}_{4}\right)\end{array}\right)$,

with $\bar{\lambda}_{k}$ are the eigenvalues in (11) calculated at the averaged state (16). The right and left eigenvector matrices are given by

$$
\mathcal{R}(\overline{\mathbf{W}})=\left(\begin{array}{cccc}
-\bar{\sigma}^{2} & \bar{r} & 1 & 1 \\
0 & \overline{r u} & \bar{\lambda}_{3} & \bar{\lambda}_{4} \\
-\bar{\sigma}^{2} \bar{c} & \overline{r c}-1 & \bar{c} & \bar{c} \\
-\bar{\lambda}_{3} \bar{\lambda}_{4} & 0 & 0 & 0
\end{array}\right),
$$

$$
\mathcal{R}^{-1}(\overline{\mathbf{W}})=\left(\begin{array}{cccc}
0 & 0 & 0 & \frac{-1}{\bar{\lambda}_{3} \bar{\lambda}_{4}} \\
\bar{c} & 0 & -1 & 0 \\
\frac{\bar{\lambda}_{4}-\overline{c r \sigma}}{2 \bar{\sigma}} & \frac{-1}{2 \bar{\sigma}} & \bar{r} & \frac{-\bar{\sigma}}{2 \bar{\lambda}_{3}} \\
-\frac{\bar{\lambda}_{3}+\overline{c r \sigma}}{2 \bar{\sigma}} & \frac{1}{2 \bar{\sigma}} & \bar{r} & \frac{\bar{\sigma}}{2 \bar{\lambda}_{4}}
\end{array}\right),
$$


where $\bar{\sigma}=\sqrt{g \bar{h}}$ is the wave speed and $\bar{r}=\frac{\rho_{s}-\rho_{w}}{2 \bar{\rho}}$ is a density ratio. Using the above matrices, the averaged state $\mathbf{W}_{i+1 / 2}^{n}$ can be easily obtained from the predictor stage in 15. Once these states are computed, the solution state $\mathbf{W}_{i}^{n+1}$ is recovered by using the corrector stage in (15).

In our FV method, the discretization of the source term $\mathbf{S}$ in (15) is carried out such that the scheme is well balanced with discretization of the flux gradients using the concept of C-property, see for example [5]. Here, a numerical scheme is said to satisfy the stillwater equilibrium (C-property) for the equations (9) if the condition

$$
\begin{gathered}
E-D=0, \quad u=0, \quad B^{n}=\bar{B}(x), \\
h+B=C_{1}, \quad \rho=C_{2},
\end{gathered}
$$

holds for stationary flows at rest. In the above, $C_{1}$ and $C_{2}$ are nonnegative constants. Therefore, the treatment of source terms in (15) is reconstructed such that the condition (17) is preserved at the discretized level. Hence, the source terms in the corrector stage (15) are discretized as

$$
\begin{aligned}
& \mathbf{S}_{i}^{n}=\left(\begin{array}{c}
0 \\
-g \frac{h_{i+1 / 2}^{n}+h_{i-1 / 2}^{n}}{2} \frac{B_{i+1}^{n}-B_{i-1}^{n}}{2 \Delta x}-\frac{\left(\rho_{s}-\rho_{w}\right)}{\frac{\rho_{i}^{n}}{n}} g\left(\frac{h_{i+1 / 2}^{n}+h_{i-1 / 2}^{n}}{2}\right)^{2} \frac{c_{i+1}^{n}-c_{i-1}^{n}}{2 \Delta x} \\
0 \\
0
\end{array}\right), \\
& \mathbf{Q}(\mathbf{W})=\left(\begin{array}{c}
\frac{E-D}{1-p^{n}} \\
-\frac{\left(\rho_{0}-\rho\right)(E-D) u_{i}^{n}}{\rho(1-p)}-g \frac{h_{l+1 / 2}+h_{i-1 / 2}^{n}}{2} S_{f} \\
E-D \\
-\frac{E-D}{1-p}
\end{array}\right)
\end{aligned}
$$$$
\int_{z_{j-1 / 2}}^{z_{j+1 / 2}} \frac{\partial B}{\partial t} d z+\int_{z_{j-1 / 2}}^{z_{j+1 / 2}} \frac{\partial B}{\partial z} d z=\int_{z_{j-1 / 2}}^{z_{j+1 / 2}} S(B) d z
$$
rect transport of bed-load by water flows. A numerical scheme which does not satisfy the C-property may produce nonphysical oscillations in the bed resulting in a poor resolution of shocks and instability problems.

\subsection{Bed Disctization}

To implement the interface-tracking tool 2 dimensional discritisation is required - in order to reduce computational expense the bed is only discretized to a safe depth. This means that the bed is broken into cells as shown in Figure 2.

The Bed assumed to be stationary and therefor fluxes in the $\mathrm{x}$ direction are assumed $=0$. Though in the continuous form as shown (3) all flux from the perspective of the bed could be described with the source term, the boundary fluxes become very useful when control volumes are eroded or overfilled. The conservative form is as shown

$\frac{\partial B}{\partial t}+\frac{\partial F(B)}{\partial z}=S(B)$
Using the Green's formula and using Euler scheme for the time derivative we obtain

$$
B_{i, j}^{n+1}=B_{i, j}^{n}-\frac{\Delta t}{\Delta z}\left(\mathcal{F}_{i, j+1 / 2}^{n}-\mathcal{F}_{i, j-1 / 2}^{n}\right)+\Delta t S_{i, j} .
$$

We can set $\Delta z=1$ as $\mathrm{h}$ is independent of $\mathrm{z}$ and $\mathrm{h}$ is assumed uniform accross the control volume The Source term is know to us as

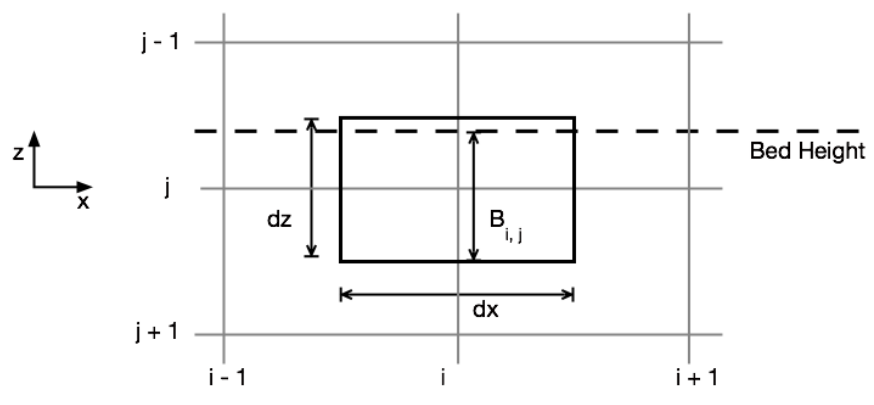

Figure 2: Control Volume $\left(C V_{i, j}\right)$ of bed cell at $\mathrm{i}, \mathrm{j}$

Then integrating 18 over the control volume we obtain:

$S_{i, j}=-\frac{E_{i, j}^{n}-D_{i, j}^{n}}{1-p_{i, j}^{n}}$.

Now consider the case where the source term (net erosion or deposition) in the cell exceeds the bounds of the cell. Boundary fluxes can be expressed as

$$
\mathcal{F}_{i, j-1 / 2}^{n}= \begin{cases}B_{i, j-1}^{n}+S_{i, j-1}^{n}, & \text { if } B_{i, j-1}^{n}+S_{i, j-1}^{n}<0, \\ 0, & \text { elsewhere. }\end{cases}
$$

If the $C V_{j+1}$ is depleted, any further erosion will occur in the cell below $\left(C V_{j}\right)$ as shown in Equation 22. When the cell beneath $C V_{j}$ is overfilled

$$
\mathcal{F}_{i, j+1 / 2}^{n}= \begin{cases}S_{i, j-1}^{n}-B_{i, j+1}^{n}, & \text { if } B_{i, j+1}^{n}+S_{i, j+1}^{n}>=d z \\ 0, & \text { elsewhere. }\end{cases}
$$


Then $C V_{j}$ begins to fill. So long as $|S|<d z$ the implementation of this method only requires evaluation of the 3 cells closest to the bed height, thus reducing computational runtime.

To implement this we use a test bed height $B_{*} i, j^{n+1}=B_{i, j}^{n}+\delta B_{* i, j}^{n+1}$. For the test case we assume an infinite cell height thus the boundary fluxes are zero, so

$\Delta B_{* i, j}=B_{* i, j}^{n+1}-B_{* i, j}^{n}=\Delta t\left(\frac{E_{i, j}^{n}-D_{i, j}^{n}}{1-p_{i, j}^{n}}\right)$,

that tested for the three possible cases (overfill, depletion and internal flux).

Notice that the considered suspended sediment transport system (9) have to be solved in a spatial domain and for a time interval endowed with given initial and boundary conditions. In practice, these conditions are problem dependent and their discussion is postponed for section 5 where numerical examples will be presented. To handle sediment mixing between the bed and flow, two assumptions where made: Firstly so long as $\Delta x$ was small enough, the cell could be equated to and expressed as the sum of the heights of the sediments types inside it. We introduce a third subscript $\mathrm{k}(1-m)$ to denote the sediment type, thus:

$B_{i, j}=b_{i, j, 1}+b_{i, j, 2}+\ldots b_{i, j, m}$,

where $b_{i, j, m}$ is the corresponding height of sediment m contained within the cell i,j. Secondly that the cell mixture is homogeneous and that the erosion rates from the cell are proportional to the sediment composition in the cell such that

$E_{i, j}=\frac{b_{i, j, 1}}{B_{i, j}} E_{i, j, 1}+\frac{b_{i, j, 2}}{B_{i, j}} E_{i, j, 2}+\ldots \frac{b_{i, j, m}}{B_{i, j}} E_{i, j, m}$,

where $E_{i, j, 1}$ is the erosion rate of $C V_{i, j}$ if it only contained only type 1 sediment.

To enable this the theoretical free bed changes for each sediment type are calculated $\Delta b_{i, j, 1}, \Delta b_{i, j, k}$ etc. and applied to the sediment heights as a test, such that:

$b_{i, j, k *}^{n+1}=b_{i, j, k}^{n}+\Delta b_{i, j, k}=b_{i, j, k}^{n}+\Delta t\left(\frac{E_{i, j, k}-D_{i, j, l}}{1-p_{i, j, k}}\right)$

Where $\delta b_{i, j *}^{-}$is the sum of all the negative and $\delta b_{i, j *}^{+}$ is the sum of all the positive $\delta b_{i, j, k}$ sediment fluxes in the cell. The four possible cases were: dell depletion where the cell is entirely eroded, the cell below becomes the active cell. Cell overfill where the cell is overfilled, the cell above becomes the active cell. Cell armouring occurs when all but one sediment types are eroded, thus the cell holds out against depletion, and the active cell stops cells below from being eroded. Intracell flux occurs when erosion and deposition rates in the cell do not exceed the bounds of the cell.

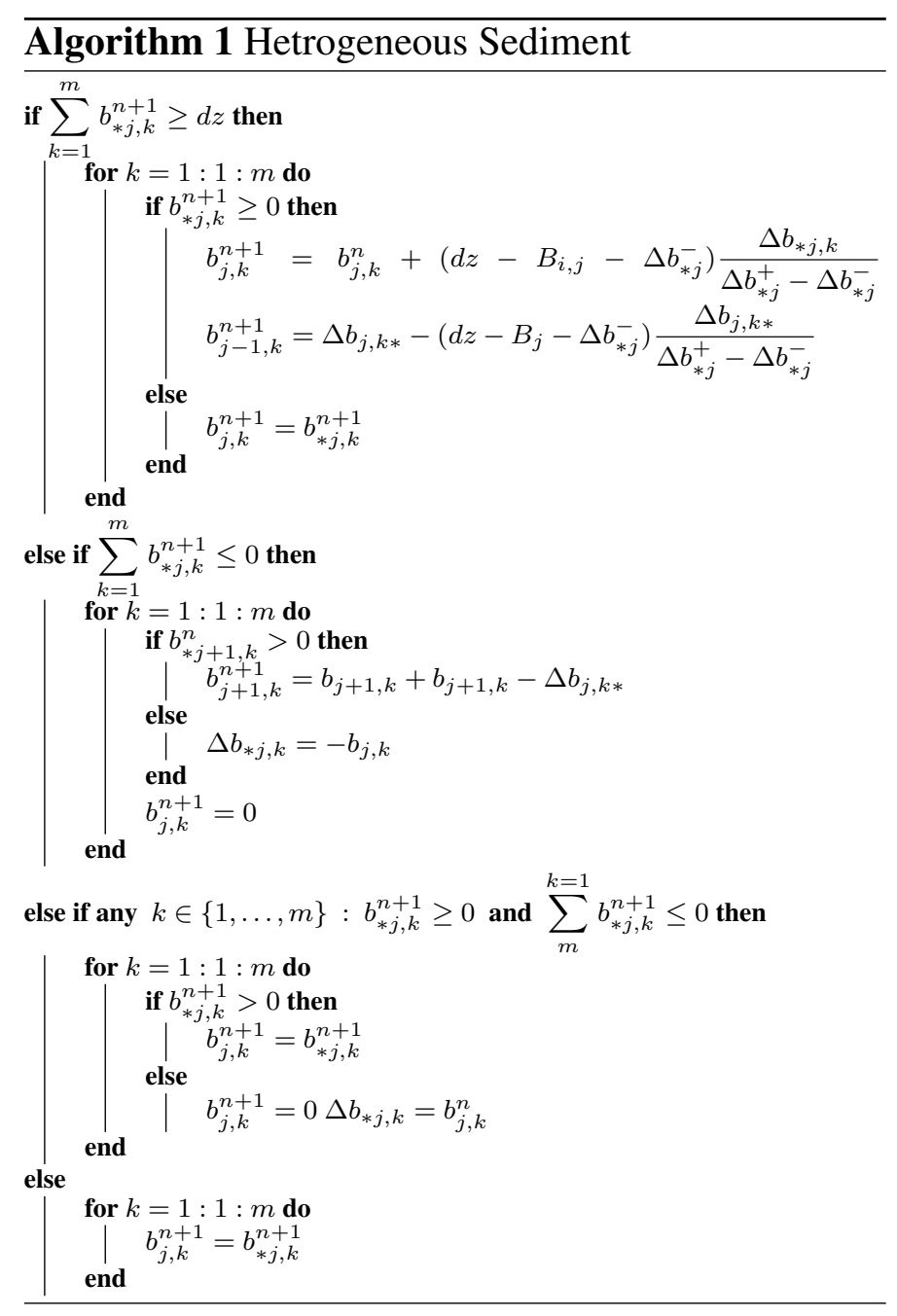

\subsection{Algorithm Integration}

As beyond the source terms, the code runs on single depth averaged concentration of suspended sediment $c$, the source terms must return a single value of $c$, such that

$c_{i}=\sum_{n}^{k=1} c_{i, k}$

It is also required that values for effective porosity, and effective density (of sediment bed) are returned to satisfy the conservation of momentum as used in the main calculation. In line with the methods used by Terzaghi et al. [18] a weighted average is used:

$p_{i}=\sum_{n}^{k=1} \frac{p_{k} z_{i, j, k}}{z_{i, j}}, \quad \rho_{s e d, i}=\sum_{n}^{k=1} \frac{\rho_{k} z_{i, j, k}}{z_{i, j}}$. 
When simulation the case of armouring, inappropriate discretization of the bed will have a direct effect on the morphology. A lower limit on discretization is set by the largest particle size inside it - $d$ in an homogeneous sediment. Often erosion and deposition calculations (including the near field ones presented in this paper) $d \sim d_{50}$ so it is reasonable therefore to set the minimum at around 10 times $d_{50}$ (for a well graded soil). Therefore for multilayer discretization where armouring is possible the following constraint is applied:

\section{$\Delta z \geq 10 d_{50}$.}

The algorithm is included at the erosion and deposition calculations. The general method uses a corrector and predictor step, and the bed calculations are designed to mirror this. Though it is included in the predictor step, the data values are not passed back or saved until the corrector step. In order to ensure total accuracy correction is finally checked against total sediment calculated in the main path.

\section{RESULTS AND APPLICATIONS}

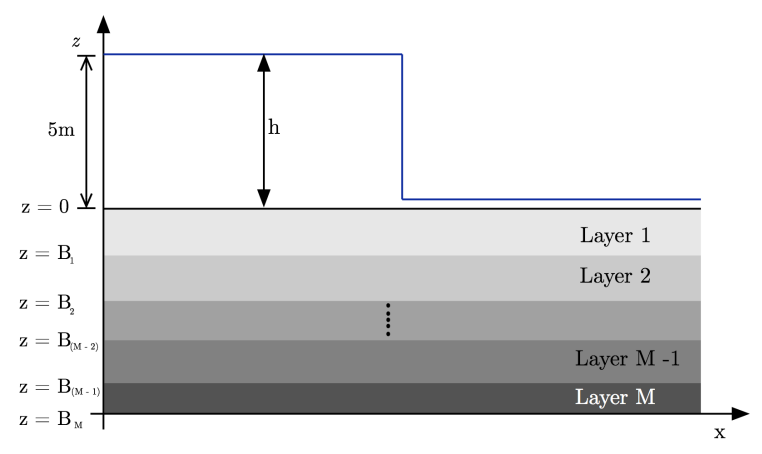

(a) Multilayer domain

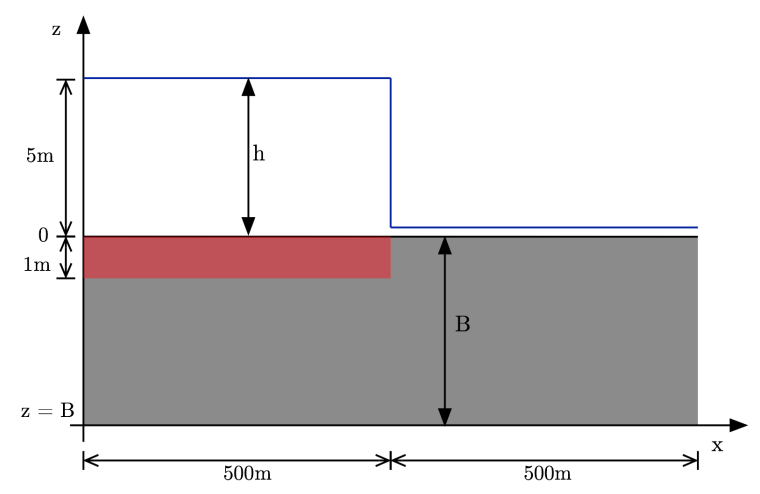

(b) Single layer domain

Figure 3: Sketches of far-field simulation domains

In this section we present numerical results for several test cases of hydraulics over multi-layered erodible beds. The emphasis in the current work is on sediment transport in dam-break problems and the main goals of this section are to illustrate the numerical performance of the techniques described above and to verify numerically their capability to solve dam-break flows over multi-layered erodible sediment beds. In all the computations reported herein, the Courant number CFL is set to 0.85 and the time stepsize $\Delta t$ is adjusted at each step according to the stability condition

Table 1: Sediment parameters used in the multi-layered simulations $[6,19,17]$.

\begin{tabular}{lclccc} 
Material & $d[\mathrm{~mm}]$ & $p$ & $\varphi$ & $\omega$ & $\rho\left[\mathrm{kg} / \mathrm{m}^{3}\right]$ \\
\hline Sand 1 & 0.0625 & 0.5 & 0.015 & 0.00014 & 1650 \\
Sand 2 & 0.25 & 0.35 & 0.015 & 0.001 & 1650 \\
Sand 3 & 0.20 & 0.4 & 0.015 & 0.0015 & 1600 \\
Sand 4 & 1 & 0.25 & 0.015 & 0.002 & 1520 \\
Clay & 0.07 & 0.51 & 0.012 & 0.0001 & 1200 \\
Silt & 0.016 & 0.55 & 0.01 & 0.0001 & 1250 \\
Pearls & 6.1 & 0.4 & 0.000015 & 0.0001 & 1048 \\
\hline
\end{tabular}

In all computations the Courant number CFL is used to vary the time step $\Delta t$, according to the stability condition

$$
\Delta t=\mathrm{CFL} \frac{\Delta x}{\max _{k=1, \ldots, 4}\left(\left|\lambda_{k}^{n}\right|\right)},
$$

where $\lambda_{k}(k=1, \ldots, 4)$ are the four eigenvalues of the sediment transport system in (11). Here, we consider the dam-break problem in a rectangular channel with a bottom initially assumed to be flat. The channel is of length $L$ and the initial conditions are given by

$$
\begin{array}{r}
h(x, 0)=\left\{\begin{array}{lll}
h_{l}, & \text { if } & x \leq L / 2, \\
h_{r}, & \text { if } & x>L / 2,
\end{array}\right. \\
u(x, 0)=u_{0}, \quad c(x, 0)=c_{0} .
\end{array}
$$

At $t=0$ the dam collapses and the flow problem consists of a shock wave travelling downstream and a rarefaction wave traveling upstream. This class of dambreak problems has been widely studied in the literature for fixed and erodible beds but using only singlelayer models, see for example [10,4] In our study we consider the multi-layered erodible beds formed with different superposed soils and the selected values for some of these materials used in our simulations are summarized in Table 2. We present numerical results for three cass of dam-break simulations including: the dam-break over single-layer bed to compare our results to experimental data; a comparison between results obtained for dam-break over multi-layered and graded beds; and modelling of sediment tracking for the dam-break over a composite bed.

\subsection{Verification test examples}

We first verify the proposed finite volume method against experimental measurements for a problem of dam-break over a single-layer erodible bed as reported in [11]. Here the bed formed by artificial spherical pearls the properties of which are listed in Table 


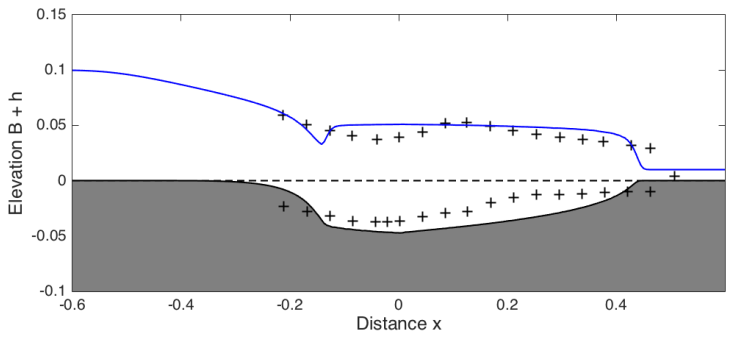

(a) A comparison of results at $t=0.505 \mathrm{~s}$

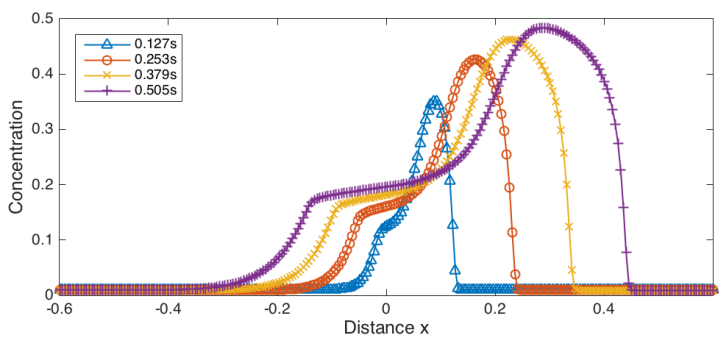

(b) Concentration profile

Figure 4: A comparison of results from the experiment and simulation results

2. The channel length is set to $L=0.6 \mathrm{~m}$ and initial conditions are given by (31) with $h_{l}=0.1 \mathrm{~m}$, $h_{r}=0.05 \mathrm{~m}, u_{0}=0 \mathrm{~m} / \mathrm{c}$ and $c_{0}=0$. The computational domain is discretized into 240 cells and the obtained results for the bed profile and water height at time $t=0.505 s$ are compared in Figure 4(a) to experimental measurements from [11]. As it can be seen from this figure there is a good agreement between the numerical results obtained using our approach and the experiments. The hydraulic jump is well captured by the proposed method and the erosion is also well resolved and it flows the experimental profile. The time evolution and concentration solution is presented in Figure 4(a) for this test problem. It is evident that the computed concentration solutions are free from any non-physical oscillations and shocks are well detected by the proposed finite volume method.

\begin{tabular}{|c|c|c|c|c|c|c|}
\hline & \multicolumn{5}{|c|}{ Horizontal discretization } \\
\hline & & $\Delta x=2.33 \mathrm{~m}$ & $\Delta x=2.86 \mathrm{~m}$ & $\Delta x=2.50 \mathrm{~m}$ & $\Delta x=2.22 \mathrm{~m}$ & $\Delta x=2.00 \mathrm{~m}$ \\
\hline \multirow{10}{*}{ 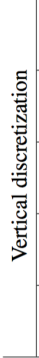 } & \multirow{2}{*}{$\Delta z=0.3 \mathrm{~m}$} & $212.69 \mathrm{~s}$ & $277.93 \mathrm{~s}$ & $362.58 \mathrm{~s}$ & $457.30 \mathrm{~s}$ & $566.69 \mathrm{~s}$ \\
\hline & & $8.73 \%$ & $6.97 \%$ & $3.54 \%$ & $1.58 \%$ & $0.44 \%$ \\
\hline & \multirow{2}{*}{$\Delta z=0.24 \mathrm{~m}$} & $206.89 \mathrm{~s}$ & $277.54 \mathrm{~s}$ & $361.94 \mathrm{~s}$ & $455.62 \mathrm{~s}$ & $564.05 \mathrm{~s}$ \\
\hline & & $9.29 \%$ & $5.97 \%$ & $3.65 \%$ & $1.16 \%$ & $0.27 \%$ \\
\hline & \multirow{2}{*}{$\Delta z=0.20 \mathrm{~m}$} & $208.03 \mathrm{~s}$ & $280.28 \mathrm{~s}$ & $362.74 \mathrm{~s}$ & $458.23 \mathrm{~s}$ & $567.38 \mathrm{~s}$ \\
\hline & & $7.96 \%$ & $5.75 \%$ & $3.46 \%$ & $1.13 \%$ & $0.08 \%$ \\
\hline & \multirow{2}{*}{$\Delta z=0.17 \mathrm{~m}$} & $207.46 \mathrm{~s}$ & $279.48 \mathrm{~s}$ & $364.12 \mathrm{~s}$ & $460.65 \mathrm{~s}$ & $565.50 \mathrm{~s}$ \\
\hline & & $8.30 \%$ & $6.47 \%$ & $3.46 \%$ & $2.13 \%$ & $0.30 \%$ \\
\hline & \multirow{2}{*}{$\Delta z=0.15 \mathrm{~m}$} & $207.97 \mathrm{~s}$ & $279.63 \mathrm{~s}$ & $364.12 \mathrm{~s}$ & $460.65 \mathrm{~s}$ & $570.63 \mathrm{~s}$ \\
\hline & & $7.66 \%$ & $5.75 \%$ & $3.79 \%$ & $1.60 \%$ & - \\
\hline
\end{tabular}

Table 2: CPU times and errors in the bed profiles using different spatial discretization steps $\Delta x$ and $\Delta z$.

Next we examine the convergence of the proposed techniques with respect to the spatial discretizations. Here we consider the problem of a dam-break over single-layered bed studied in [19] among others. The length of computational domain is $L=1000 \mathrm{~m}$ and initial conditions are given by (31) with $h_{l}=5 \mathrm{~m}$, $h_{r}=0.01 \mathrm{~m}, u_{0}=0 \mathrm{~m} / \mathrm{c}$ and $c_{0}=0.0001$. The bed depth is of $2 m$ and it is formed by Sand 2 and the associated properties can be found in Table 2. The purpose of this test example is to check the grid convergence in our finite volume method. Since this problem has no analytical solution to be used to quantify the errors in our computations, we have used reference solutions computed on a very fine grid using $\Delta x=2 \mathrm{~m}$ and $\Delta z=0.15 \mathrm{~m}$. In all our simulations we vary the spatial discretization steps $\Delta x$ and $\Delta z$ and we compute the relative errors in the bed profile compared to the reference solution. In Table 2 we summarize the errors (in \%) and the CPU times (in seconds) for different sizes of steps $\Delta x$ and $\Delta z$. It is clear that increasing the number of gridpoints in the horizontal discretization or decreasing the step in the vertical discretization results in an improvement in the relative errors at the cost of an increase the CPU times. An important remark concerning this convergence study is related to the fact that for the considered sediment transport, the vertical discretization of the bed does not affect the overall accuracy of our finite volume method. The spatial accuracy of the proposed method is sensible only to the horizontal discretization step $\Delta x$. It should also be stressed that refining the vertical discretization retains the computational cost at its minimum. Similar remarks have been observed for numerical simulations, not reported here, without vertical discretization.

\subsection{Dam-break over multi-layered bed}

In this test example we solve a problem of dam-break over a multi-layered bed formed with five layers of superposed soils. As in the previous test examples, the length of computational domain is $L=1000 \mathrm{~m}$ and initial conditions are given by (31) with $h_{l}=5 \mathrm{~m}$, $h_{r}=0.01 \mathrm{~m}, u_{0}=0 \mathrm{~m} / \mathrm{c}$ and $c_{0}=0.0001$. The depth of the bed is $2 m$ and initially it is formed by

$$
B(x, z)=\left\{\begin{array}{lll}
\text { Sand } 1 & \text { if } & -0.5 m \leq z<0 \mathrm{~m}, \\
\text { Sand } 2 & \text { if } & -1.0 m \leq z<-0.5 \mathrm{~m}, \\
\text { Sand } 3 & \text { if } & -1.5 m \leq z<-1.0 \mathrm{~m}, \\
\text { Clay } & \text { if } & -2.0 \mathrm{~m} \leq z<-1.5 \mathrm{~m}, \\
\text { Silt } & \text { if } & z<-2.0 \mathrm{~m},
\end{array}\right.
$$

with the associated properties given in Table 1. Note that the density and porosity normally vary as a function of depth and often contain discrete layers of sediments, see for example [15]. For all the results presented for this test example the spatial discretizations are carried out using $\Delta x=2.22 \mathrm{~m}$ and $\Delta z=0.15 \mathrm{~m}$ and obtained solutions for the bed, the water freesurface and the sediment concentration are illustrated at four different times $t=5 \mathrm{~s}, 10 \mathrm{~s}, 15 \mathrm{~s}$ and $20 \mathrm{~s}$. Note that this spatial discretization is selected based on the balance between the accuracy and computational cost reported in Table 2 .

In Figure 5 we present the time evolution of the bed, the water free-surface and the sediment concen- 
tration. Different colours have been used in these plots to distinguish between the layers in the bed. Darker colours have been used for deeper sediment layers and its associated concentration. It is evident from the presented results that dam-break flows over 5-layered erodible sediment beds are highly affected by the sediment properties of the soil used in its formation, such that the finer the sediment the greater effects bed mobility will have. It is worth remarking that an increase of the averaged diameter of the sediment particles $d$ yields to an increase in the deposition coefficient $\omega$ which is used in the deposition term (4). Thus, a weak deposition effect is expected for small sediment particles. As can be observed from these results, the erosion effects on the bed are clearly visible for small values of the bed sediment particle size. The variation of the bed sediment particle size creates a very active sediment exchange between the water flow and the bed loads, and also produces a sharp spatial gradient of sediment concentration, which justifies its incorporation in the momentum equation (1). Apparently, the overall flow and sediment features for this example are preserved with no spurious oscillations appearing in the results obtained using our finite volume method. Obviously, the computed results verify the stability and the shock capturing properties of the proposed techniques.

\subsection{Dam-break over composite bed}

Our final example consists of a dam-break problem over a composite bed in which sediment tracking is examined in our simulation. This class of problem occurs mainly in tailings transport in mining, see for instance the case study of dam-break in Canada [16] and in Brazil [13]. The problem statement is formulated using the equations (1) and a configuration of the domain and bed profile is depicted in Figure 3(b). A thin layer of tailings is localized upstream of the dam and the bed is a composite of tailings and clay. As in the previous test example of dam-break in a multilayered bed, the spatial discretization is carried out using $\Delta x=2.22 m$ and $\Delta z=0.15 m$ and obtained solutions for the bed, the water free-surface and the sediment concentration are illustrated at four different times $t=5 \mathrm{~s}, 10 \mathrm{~s}, 15 \mathrm{~s}$ and $20 \mathrm{~s}$. The emphasis in this test problem is on the tracking of suspended sediment in the water flow for the considered instants.

Figure 6 exhibits the time evolution of the bed, the water free-surface and the sediment concentration obtained for the four selected instants. A different shade used to identify the thin layer of tailings in the presented results. A good numerical accuracy is required in order to capture the different phenomena present in its evolving solution. As a consequence, the later sediment transport problem is more difficult to handle; the results shown here illustrate the robustness of the proposed numerical techniques. As can be seen, the suspended sediment is well tracked and the location of its concentration is also well detected using our finite volume method. For the considered sediment characteristics, no deposition effects on the bed have been observed in it. As can be observed from the results in Figure 6, the propagation of the water flow over the movable bottom has been accurately captured by our finite volume method. During the flow propagation, an hydraulic jump is formed near the initial dam site and propagates upstream. The upper front of the hump travels faster than the lower base so that the propagating bed-load creates a shock at the front after a certain time.

As can be observed from these results, the dambreak flow over movable bed can build up a heavily concentrated wavefront, which is bounded by the wave forefront and a contact discontinuity of the sediment transport, and which depresses in the long run. The bed mobility can strongly modify the water freesurface profiles, and may have considerable implications for flood predictions. As in the previous simulations, a hydraulic jump in the water free-surface is initially formed around the dam site, depresses progressively as it propagates upstream, and eventually disappears. It is evident that the movable bed can be significantly scoured and the dimensions of the scour hole are of similar order of magnitude to those of the water flow itself. Therefore the rate of bed deformation is not negligible compared to that of the flow change, characterizing the need for coupled modelling of the strongly interacting flow-sediment-morphology system as the one considered in the present work. The proposed finite volume method performs well for this test problem since it does not diffuse the moving fronts and no spurious oscillations have been observed when the water flows over the movable bed. The proposed techniques also makes tracking easy, as well as allowing for different materials to be eroded according to their properties, their suspended concentration and final location are recorded.

\section{CONCLUSIONS}

In this paper we have studied the accuracy and efficiency of a class of finite volume methods which are investigated for numerical solution of morphodynamic problems in multi-layered beds. The governing equations include an hydraulic part described by the shallow water equations, and a sediment part described by the transport and suspended equations. To allow vertical exchange between the bed layers we propose an equation of balance laws for the bed elevation. The proposed finite volume method consists of two stages which can be viewed as a predictorcorrector procedure. In the first stage, the scheme reconstructs the numerical fluxes using the sign matrix of the flux Jacobian in the morphodynamic equations. This stage results in an upwind discretization of the characteristic variables and avoids the singularity that may occur in the Jacobian matrix for the conservative 

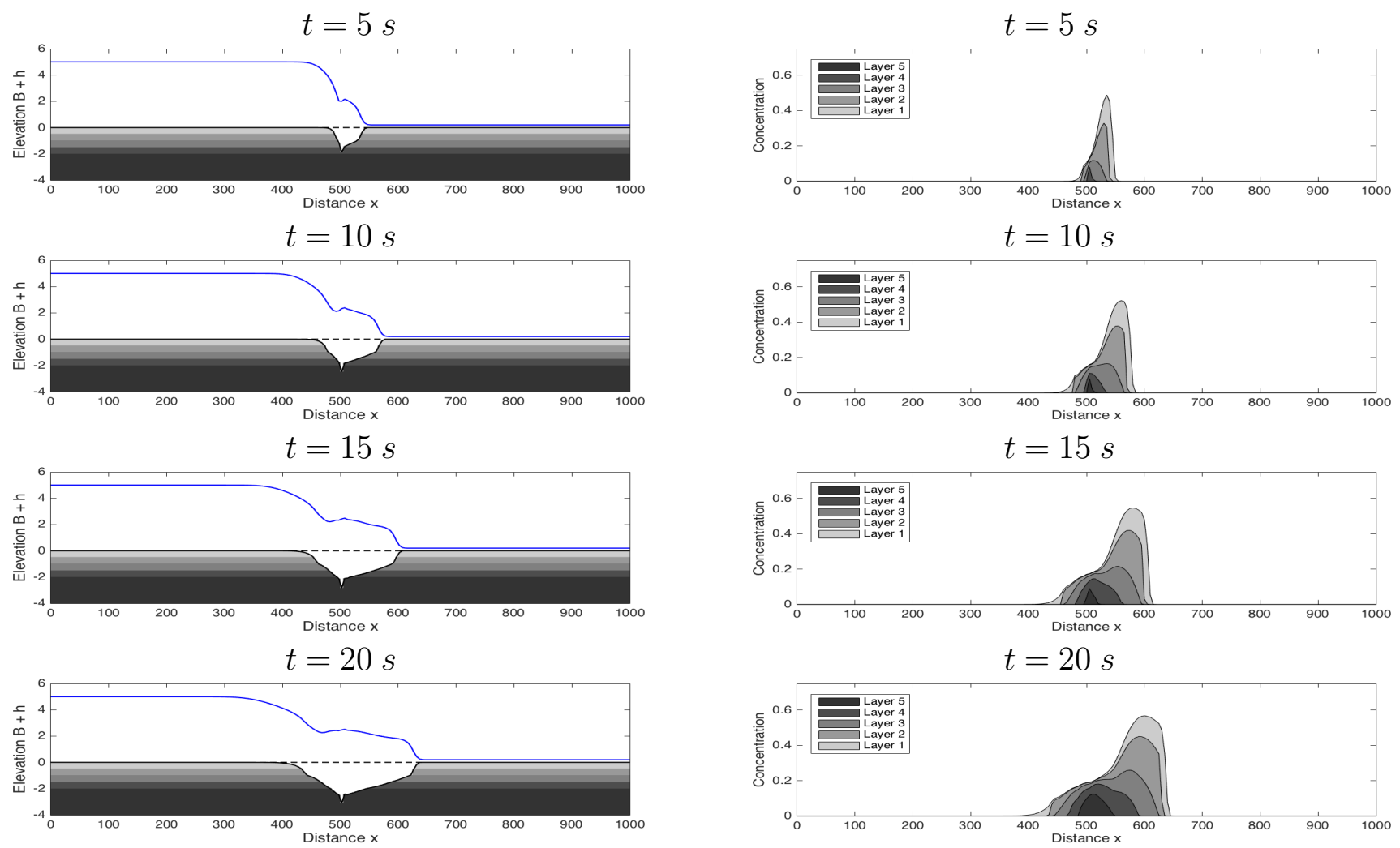

Figure 5: Bed and water free-surface (left column) and sediment concentration (right column) at four different instants for the problem of dam-break over 5-layered bed.
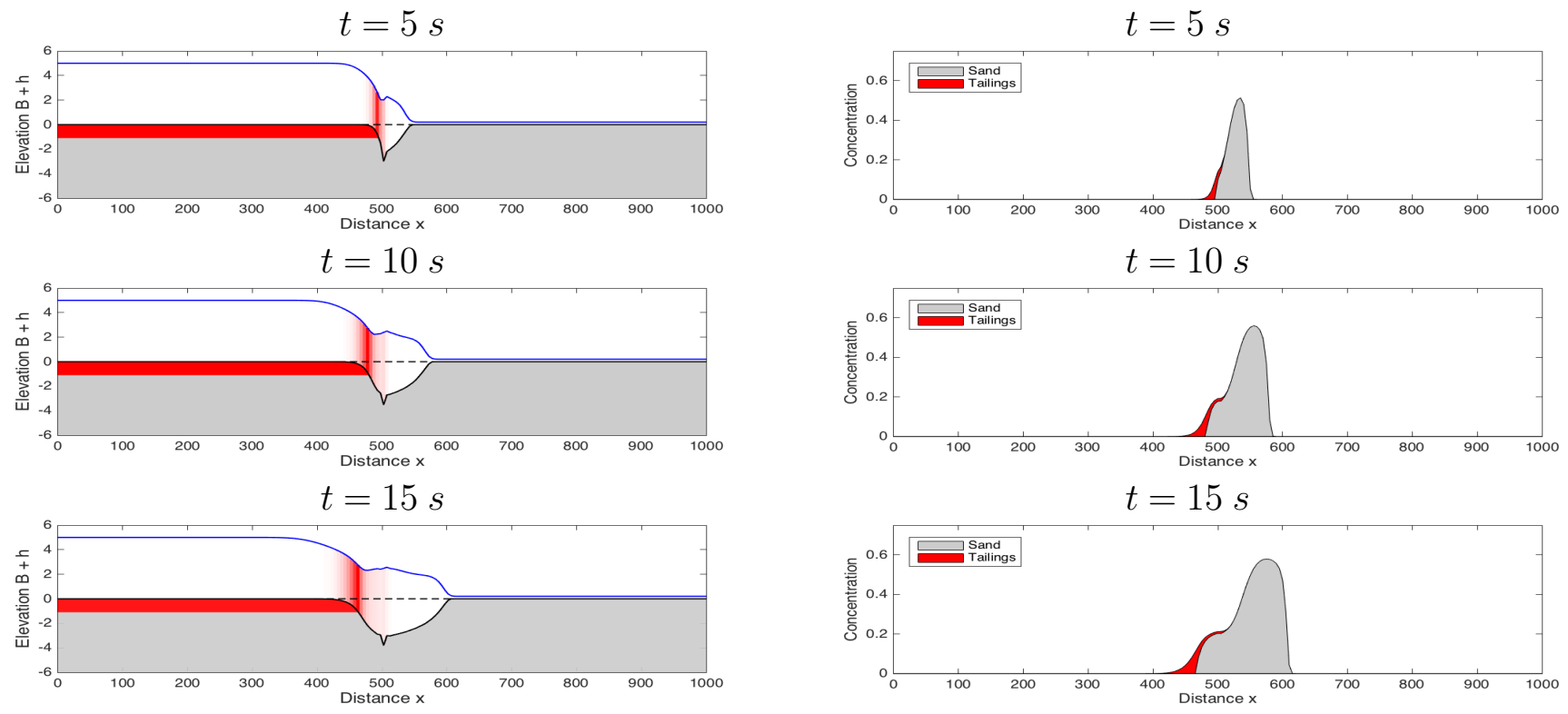

$t=15 \mathrm{~s}$
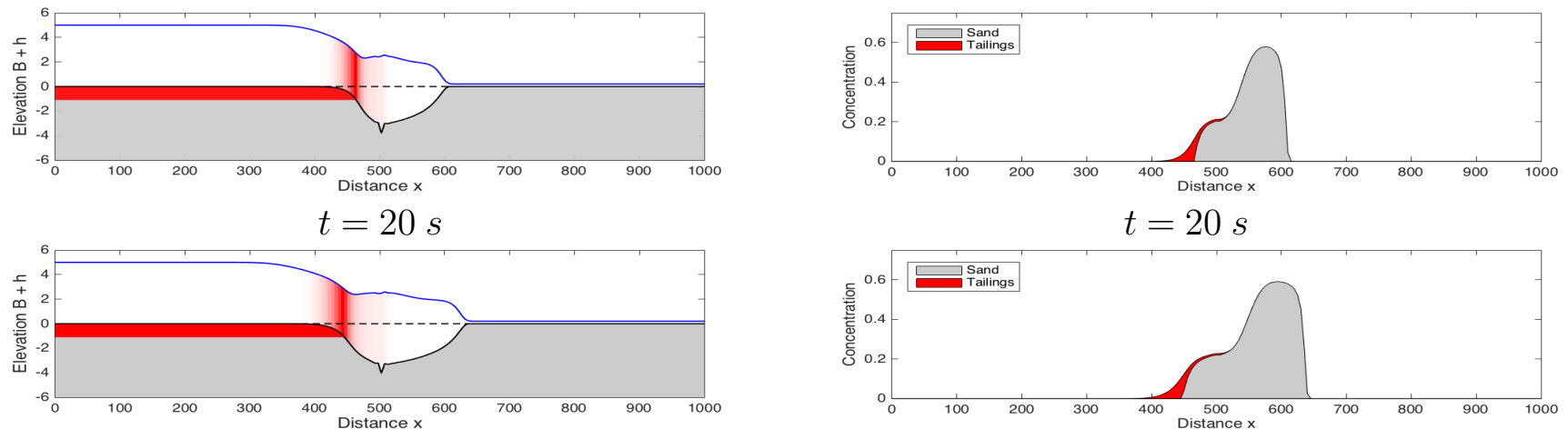

Figure 6: Bed and water free-surface (left column) and sediment concentration (right column) at four different instants for the problem of dam-break over composite bed.

form of the morphodynamic equations. In the second stage, the solution is updated using a special treatment of the bed bottom in order to obtain a well-balanced discretization of the flux gradient and the source term. In addition a conservative vertical discretization has been carried out for the multi-layered bed to account for different soil properties forming the bed.

The proposed techniques have been numerically examined for several test examples in dam-break problems over multi-layered erodible beds. We have 
compared the numerical results obtained for singlelayer bed to experimental measurements. The method has also been applied to dam-break over 5-layered bed and over a composite bed. In most cases, the proposed methods have exhibited accurate prediction of both, the free surface and the bed-load with correct conservation characteristics and stable representation of free-surface response to the movable bed. The results make it promising to be applicable also to real situations where, beyond the many sources of complexity, there is a more severe demand for accuracy in predicting the morphological evolution, which must be performed for long time. Future work will concentrate on developing efficient time integration schemes for the finite volume discretization and extension of these approaches to morphodynamic flow problems in two space dimensions.

\section{REFERENCES}

[1] M.B. Abbott. Computational hydraulics: Elements of the theory of free surface flows. London: Fearon-Pitman Publishers, 1979.

[2] F. Benkhaldoun, S. Sahmim, and M. Seaïd. "A two-dimensional finite volume morphodynamic model on unstructured triangular grids." In: International Journal of Numerical MEthods in Fluids 63 (2010), pp. 1296-1327.

[3] F. Benkhaldoun, S. Sahmim, and M. Seaïd. "Solution of the sediment transport equations using a finite volume method based on sign matrix." In: SIAM Journal of Scientific Computing 31 (2009), pp. 2866-2899.

[4] F. Benkhaldoun, S. Sari, and M. Seaid. "A fluxlimiter method for dam-break flows over erodible sediment beds." In: Applied Mathematical Modelling 36 (2008), pp. 4847-4861.

[5] A. Bermúdez and M. E. Vázquez. "Upwind methods for hyperbolic conservation laws with source terms. Computers \& Fluids". In: Flow Turbulence Combustion 23 (1994), pp. 1049 1071.

[6] Van Rijn L. C. "Unified view of sediment transport by currents and waves. $\mathrm{i}$ :Initiaion of motion, bed roughness, and bed-load transport". In: Journal of Hydraulic Engineering 113 (2007), pp. 649-667.

[7] Z. Cao and P. Carling. "Mathematical modelling of alluvial rivers: reality and myth. Part I: General overview." In: Water Maritime Engineering 154 (2002), pp. 207-220.

[8] Z. Cao and G. Pender. "Numerical modelling of alluvial rivers subject to interactive sediment mining and feeding." In: Advances in Water Resources 27 (2005), pp. 533-546.
[9] Z. Cao, G. Pender, and P. Carling. "Shallow water hydrodynamic models for hyperconcentrated sediment-laden floods over erodible bed." In: Advances in Water Resources 29 (2006), pp. 546-557.

[10] Z. Cao et al. "Computational dam-break hydraulics over erodible sediment bed." In: Journal of Hydraulic Engineering 67 (2004), pp. 149-152.

[11] D. Capart and D. L. Young. "Formation of jump by the dambreak wave over a granular bed". In: Journal of Fluid Mechanics 372 (1998), pp. $165-187$.

[12] M. Hammouch et al. "SRNH finite volume scheme with mesh adaptation for shallow water equations and variable topography". In: Physical and Chemical News 59 (2011), pp. 13-21.

[13] P. Kiernan. Brazil Dam's Failure Flooded Region With Toxis Waste, U.N. Report Says. Ed. by wsj.com. [Online; posted 25-November2015]. 2015. URL: http : / / www . wsj . com / articles / brazil - dams - failure flooded-region-with-toxic-wastes-un-report-says-1448494712.

[14] D. F. Liang, B. L. Lin, and R. A. Falconer. "A Boundary-Fitted numerical model of flood routing with shock-capturing capability". In: Journal of Hydrolics 332 (2007), pp. 447-486.

[15] T. H. McCulloh. "Mass properties of sedimentary rocks and gravimetric effects of petroleum and natural-gas reservoirsSimplified settling velocity formula for sediment particle". In: United States Geological Survey 528-A (1967). USGS Proffesional Paper.

[16] N. R. Morgenstern. Report on Mount Polley Tailings Storage Facility Breach. Ed. by Independent Expert Engineering Investigation and Review Panel. [Online; posted 30January-2015]. 2015. URL: https : / / www . mountpolleyreviewpanel.ca/sites/.

[17] W. W. Rubey. "Settleing velocity of gravel, sand, and silt particles". In: American Journal of Science 148 (1933), pp. 325-338.

[18] K. Terzaghi, R. B. Peck, and G. Mersi. Soil Mechanics in Engineering Practice. New York: John Wile \& Sons Publishers, 1996.

[19] W. Wu and S. S. Wang. "Formulas for sediment porosity and settling velocity". In: Journal of Hydraulic Engineering 132 (2006), pp. 858862. 